\title{
Diversidade de plantas alimentícias encontradas em quintais da comunidade Bom
}

\section{Lugar, Campo Maior-PI}

\author{
Diversity of food plants found in backyards of the Bom Lugar community, Campo Maior-PI \\ Diversidad de plantas alimenticias encontradas en los patios traseros de la comunidad de Bom \\ Lugar, Campo Maior-PI
}

Recebido: 30/04/2021 | Revisado: 06/05/2021 | Aceito: 14/05/2021 | Publicado: 29/05/2021

\author{
Adriana de Sousa Lima \\ ORCID: https://orcid.org/0000-0001-8420-3312 \\ Universidade Federal do Piaú, Brasil \\ E-mail: adrianbiologa@yahoo.com.br \\ Kelly Polyana Pereira dos Santos \\ ORCID: https://orcid.org/0000-0002-2830-1935 \\ Universidade Estadual do Piauí, Brasil \\ E-mail: kellypolyana@cte.uespi.br \\ Lúcia Gomes Pereira \\ ORCID: https://orcid.org/0000-0002-9646-2546 \\ Universidade Federal do Piaú, Brasil \\ E-mail: luciabotanica@yahoo.com.br \\ Roseli Farias Melo de Barros \\ ORCID: https://orcid.org/0000-0001-9767-5546 \\ Universidade Federal do Piauí, Brasil \\ E-mail: rbarros.ufpi@gmail.com
}

\begin{abstract}
Resumo
Os quintais são locais próximos às residências, de fácil acesso aos moradores e exercem um papel importante na promoção da segurança nutricional e alimentar às famílias em todo o mundo, bem como na conservação da biodiversidade. Objetivou-se realizar o levantamento das plantas alimentícias cultivadas em quintais rurais da comunidade de Bom Lugar, no município de Campo Maior/PI. Os dados foram levantados por meio de 54 entrevistas semiestruturadas com aplicação de formulários e turnê-guiada com os mantenedores dos quintais. Para análise dos dados, foi utilizada a estatística descritiva e o Índice de Diversidade de Shannon (H'). Dentre os entrevistados, a maior parte era do gênero feminino $(82,1 \%)$, casados $(69,6 \%)$ e com o ensino fundamental incompleto $(64,2 \%)$. Foram identificadas 70 espécies de uso alimentício. Solanaceae, Anacardiaceae, Annonaceae, Mythaceae e Rutaceae foram as famílias mais representativas em número de espécies. A espécie Anacardium occidentale L. obteve destaque nos quintais por conta do seu alto valor econômico, assim como Manihot esculenta Crantz. O índice de Diversidade de Shannon foi de $\left(\mathrm{H}^{\prime}\right)=3,703$. A maior parte das plantas encontradas cultivadas era frutíferas, seguidas por hortaliças e espécies condimentares. As plantas exóticas se sobressaíram em relação às plantas nativas. Os quintais de Bom Lugar são fontes de nutrientes e renda para os moradores garantindo assim a subsistência e a segurança alimentar.
\end{abstract}

Palavras-chave: Diversidade; Etnobotânica; Quintais Produtivos.

\begin{abstract}
Backyards are places close to homes, easily accessible to residents and play an important role in promoting nutritional and food security for families around the world, as well as in the conservation of biodiversity. The objective was to carry out a survey of food plants grown in rural backyards in the community of Bom Lugar, in the municipality of Campo Maior / PI. The data were collected through 54 semi-structured interviews with the application of forms and a guided tour with the backyard keepers. For data analysis, descriptive statistics and the Shannon Diversity Index ( $\left.H^{\prime}\right)$ were used. Among the interviewees, most were female $(82.1 \%)$, married $(69.6 \%)$ and with incomplete primary education (64.2\%). 70 species of food use were identified. Solanaceae, Anacardiaceae, Annonaceae, Mythaceae and Rutaceae were the most representative families in number of species. The species Anacardium occidentale L. was highlighted in backyards due to its high economic value, as well as Manihot esculenta Crantz. Shannon's Diversity Index was $\left(\mathrm{H}^{\prime}\right)=3.703$. Most of the plants found cultivated were fruitful, followed by vegetables and condiment species. Exotic plants stood out compared to native plants. Bom Lugar's backyards are a source of nutrients and income for residents, thus ensuring subsistence and food security.
\end{abstract}

Keywords: Diversity; Ethnobotany; Productive Backyards. 


\begin{abstract}
Resumen
Los patios traseros son lugares cercanos a los hogares, de fácil acceso para los residentes y juegan un papel importante en la promoción de la seguridad nutricional y alimentaria para las familias de todo el mundo, así como en la conservación de la biodiversidad. El objetivo fue realizar un relevamiento de plantas alimenticias cultivadas en patios rurales en la comunidad de Bom Lugar, en el municipio de Campo Maior / PI. Los datos fueron recolectados a través de 54 entrevistas semiestructuradas con la aplicación de formularios y una visita guiada con los cuidadores del patio trasero. Para el análisis de datos, se utilizaron estadísticas descriptivas y el índice de diversidad de Shannon (H'). Entre los entrevistados, la mayoría eran mujeres $(82,1 \%)$, casadas $(69,6 \%)$ y con educación primaria incompleta $(64,2 \%)$. Se identificaron 70 especies de uso alimentario. Solanaceae, Anacardiaceae, Annonaceae, Mythaceae y Rutaceae fueron las familias más representativas en número de especies. La especie Anacardium occidentale L. se destacó en patios traseros por su alto valor económico, así como Manihot esculenta Crantz. El índice de diversidad de Shannon fue $\left(\mathrm{H}^{\prime}\right)=3.703$. La mayoría de las plantas que se encontraron cultivadas fueron fructíferas, seguidas de las hortalizas y las especies de condimentos. Las plantas exóticas se destacaron en comparación con las plantas nativas. Los patios traseros de Bom Lugar son fuente de nutrientes e ingresos para los residentes, lo que garantiza la subsistencia y la seguridad alimentaria.
\end{abstract}

Palabras clave: Diversidad; Etnobotánica; Patios traseros productivos.

\title{
1. Introdução
}

A alimentação consiste em um direito social e fundamental, que assegura a todo cidadão, a garantia de acesso aos alimentos em quantidade e qualidade adequadas para o atendimento pleno de suas necessidades biológicas, todos os dias, em todos os lugares e sob todas as circunstâncias (Brasil, 1988; Batista Filho, 2010). Entretanto, apesar de ser um direito assegurado pela Constituição Federal, grande parte da população brasileira e global não tem acesso à segurança alimentar e nutricional diária (FAO et al., 2019).

A segurança alimentar e nutricional, um conceito multidisciplinar por natureza, consiste no acesso físico e econômico contínuo a alimentos seguros, nutritivos e suficientes para satisfazer às suas necessidades dietéticas e preferências alimentares, a fim de favorecer uma maior qualidade de vida (FAO, 2015).

A Organização das Nações Unidas para Alimentação e Agricultura (FAO) estimou que, aproximadamente, 2 bilhões de pessoas no mundo tiveram acesso ao mínimo à alimentação para a sobrevivência no ano de 2018 , e, destes, mais de 820 milhões passaram fome (FAO et al., 2019; Castañeda-Navarrete, 2021).

Esse cenário não é exceção no Brasil, onde áreas menos antropizadas apresentam várias condições sociais desfavoráveis que, por sua vez, levam à maior proporção de insegurança alimentar (FAO-Brasil, 2015). Dados da Pesquisa de Orçamentos Familiares (POF), no período de 2017-2018, revelam que insegurança alimentar atingiu 44\% dos domicílios rurais no Brasil, sendo as regiões Nordeste e Norte as de maior incidência (IBGE, 2020).

O estado do Piauí apresenta-se como uma região crítica de insegurança alimentar (IBGE, 2020). Dados da Pesquisa de Orçamentos Familiares (POF), no período de 2017-2018, relataram que 46\% dos domicílios piauienses apresentaram algum grau de insegurança alimentar, ocupando o $12^{\circ}$ lugar nacional e, em comparação aos outros Estados do Nordeste, ocupou a segunda posição (IBGE, 2020).

Vários são os indicadores utilizados para determinar a segurança alimentar e nutricional no mundo. No Brasil, os indicadores estabelecidos se baseiam em seis dimensões, dentre as quais se destaca a produção e disponibilidade de alimentos (IBGE, 2014).

A forma de obtenção de alimentos e quais os tipos consumidos variam de acordo com a sociedade em que as populações estão inseridas. Em ambientes menos antropizados, principalmente em países de baixa renda, espaços como quintais são reconhecidos por contribuir para a segurança alimentar das famílias, o estado nutricional e a sustentabilidade ecológica (Zhang et al., 2020). Os quintais são reconhecidos como um sistema de uso de terra, localizado próximo às residências, utilizado para manejar e cultivar uma variedade de espécies úteis (Zang et al., 2020; Kumar \& Nair, 2004). 
Reconhecidamente como modelo de sustentabilidade (Kumar \& Nair, 2004), os quintais têm recebido atenção por serem importantes sistemas agrícolas e agroflorestais, garantindo o suprimento alimentar para as famílias, bem como fonte de renda para o seu sustento (Nascimento, Alves \& Molina, 2005). Nas zonas rurais, os quintais possuem uma íntima relação com o conhecimento ecológico local, sendo considerados como refúgios bioculturais (Calvet-Mir et al., 2016).

Estudos realizados em quintais urbanos e rurais de vários países reportam altos níveis de diversidade genética de plantas inter e intraespecífica, notadamente de variedades de culturas tradicionais e crioulas que estão sendo conservadas nestes ambientes (Galluzzi, Eyzaguirre \& Negri, 2010).

No Piauí, estudos etnobotânicos têm levantado as plantas alimentícias em diferentes localidades, entretanto, estas não ganham um destaque maior, sendo muitas vezes incorporadas em formas de porcentagens totais, sem uma análise mais aprofundada da classe (Aguiar \& Barros, 2012; Pereira et al., 2016; Ribeiro, Amorim \& Barros, 2020) e, considerando que os quintais são importantes espaços de abrigo para estas espécies, objetivou-se, neste estudo, descrever as plantas alimentícias encontradas nos quintais em uma comunidade rural no município de Campo Maior, Piauí.

\section{Metodologia}

\section{1 Área de Estudo}

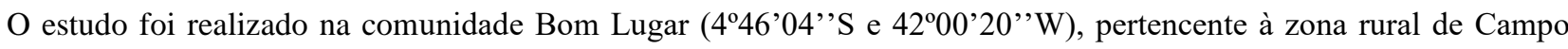
Maior (Figura 1), distando cerca de 10km da sede do município. Possui cerca de 250 pessoas, distribuídas em 70 residências. Dentre as características de infraestrutura gerais da comunidade, destacamos a presença de energia elétrica, abastecimento de água realizado por poço tubular, cacimbão e cisternas, não há coleta de lixo no local e nem rede de esgoto.

Figura 1. Localização da área de estudo, Comunidade Bom Lugar, Campo Maior/PI.
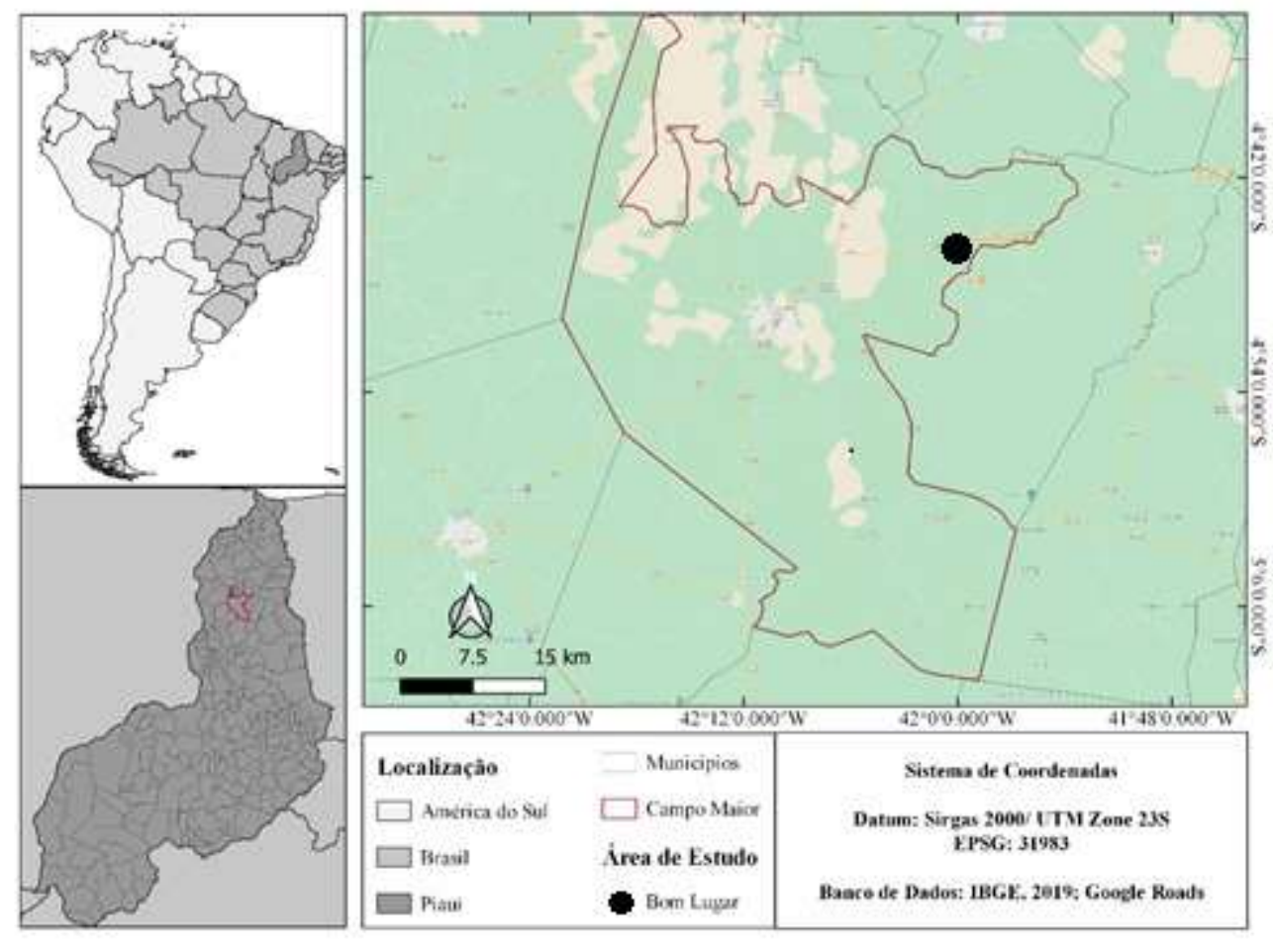

Fonte: Autores. 
Campo Maior, distando 84,4 km de Teresina, capital do Piauí, apresenta uma área total de 1.680,3 km², com uma população estimada de 46.893 habitantes para 2020; destes, 74,20\% (33.521 hab.) residem na área urbana e 25,80\% (11. 656 hab.) na zona rural (IBGE, 2010). A região apresenta clima Tropical Subúmido Seco de acordo com Köppen (As), com temperatura média de $26,8^{\circ} \mathrm{C}$ e pluviosidade média anual de $1.360 \mathrm{~mm}$ (Farias \& Castro, 2004; CEPRO, 2010). A vegetação é caracterizada por transições vegetacionais de cerrado/caatinga, cerrado e mata com vegetação caducifólia e subcaducifólia estacionais (Veloso et al., 2002).

A fim de atender os preceitos éticos da pesquisa, o trabalho foi submetido à aprovação e consubstanciação do Comitê de Ética e Pesquisa (CEP) da Universidade Federal do Piauí (UFPI), obtendo o parecer $n^{\circ} 3.070 .263$ e foi cadastrado no Sistema Nacional de Gestão do Patrimônio Genético e do Conhecimento Tradicional Associado (SISGEN), sob cadastro ${ }^{\circ}$ A16A0EO. Antes do início da coleta de dados, foi solicitada aos informantes sua assinatura no Termo de Consentimento Livre e Esclarecido/Consentimento Prévio Informado (Resolução No 510/2016 do Conselho Nacional de Saúde - CNS/Lei 13.123/15 e Decreto n8.772/16), documento no qual os informantes permitem a realização das entrevistas e aplicação dos formulários.

\subsection{Coleta de dados e Análise de dados}

Em etapa preliminar, foi feito o Rapport (Barbosa, 2007), sendo realizadas reuniões com os líderes da comunidade, com o intuito de estabelecer vínculo de confiança e conseguir resultados mais fidedignos. A coleta de dados foi conduzida com o mantenedor principal do quintal de cada domicílio, desde que fosse maior de 18 anos, por meio de entrevistas semiestruturadas (Bernard, 1988), a fim de coletar informações das espécies utilizadas na alimentação na comunidade, seu manejo, partes utilizadas e forma de consumo. Visando realizar a coleta do material botânico cultivado, foram procedidas turnês-guiadas (Bernard, 1988) para posterior identificação que foi realizada por intermédio da análise da sua morfologia, por consultas a bibliografias especializadas, envio a especialistas ou por comparação com indivíduos incorporados no herbário Graziela Barroso (TEPB) da Universidade Federal do Piauí (UFPI).

O sistema de organização adotado foi o APG IV (2016). Para a consulta da origem das plantas (nativa ou exótica), a grafia da nomenclatura científica das espécies, bem como a abreviatura dos nomes dos autores, foi utilizada o sítio da Flora Brasil (http://floradobrasil.jbrj.gov.br). Destaca-se que foram consideradas como nativas todas as plantas originadas na América do Sul e, como exóticas, as originadas fora deste território. Os dados foram agrupados (família, espécies, hábito, origem e grupos alimentares), utilizando-se de estatística descritiva para expor os dados e explicitar as espécies que compõem o rol de espécies alimentícias da comunidade. Também foi calculado o índice de Diversidade de Índice de Shannon-Wiener (Magurran, 2013) por meio do software R com auxílio da extensão RStudio (Rstudio Team, 2015).

\section{Resultados}

Dos 54 mantenedores/ entrevistados, a maior parte era do gênero feminino (82,1\%), com faixa etária entre 30 e 59 anos $(67,8 \%)$ e casadas $(69,6 \%)$ (Tabela 1). De um modo geral, os informantes apresentaram baixa escolaridade com 66,6\% com ensino fundamental incompleto. As atividades econômicas desenvolvidas na comunidade são baseadas na agricultura, pecuária, apicultura e criação de pequenos animais. A renda média mensal familiar girou em torno de um salário-mínimo. 
Tabela 1. Principais variáveis do perfil socioeconômico dos entrevistados na comunidade Bom Lugar - Campo Maior.

\begin{tabular}{|c|c|c|}
\hline Dados socioeconômicos & & Percentual (\%) \\
\hline \multirow[t]{2}{*}{ Gênero } & Feminino & 82,1 \\
\hline & Masculino & 17,9 \\
\hline \multirow[t]{3}{*}{ Faixa etária } & 18 a 29 anos & 1,8 \\
\hline & 30 a 59 anos & 67,8 \\
\hline & 60 anos ou mais & 30,4 \\
\hline \multirow[t]{6}{*}{ Escolaridade } & Sem escolarização & 14,8 \\
\hline & Ens. Fundamental Incompleto & 66,6 \\
\hline & Ens. Fundamental Completo & 9,2 \\
\hline & Ens. Médio Incompleto & 2,2 \\
\hline & Ens. Médio Completo & 5,4 \\
\hline & Ens. Superior & 1,8 \\
\hline \multirow[t]{5}{*}{ Estado civil } & Solteiro & 3,8 \\
\hline & Divorciado & 1,8 \\
\hline & Casado & 69,6 \\
\hline & União estável & 12,4 \\
\hline & Viúvo & 12,4 \\
\hline Renda mensal dominante & 1 salário mínimo & 60 \\
\hline \multirow[t]{6}{*}{ Tamanho dos quintais } & $101-500 \mathrm{~m}^{2}$ & 17,8 \\
\hline & $501-1000 \mathrm{~m}^{2}$ & 10,7 \\
\hline & $1001-1500 \mathrm{~m}^{2}$ & 16,1 \\
\hline & $15001-2000 \mathrm{~m}^{2}$ & 3,6 \\
\hline & $2000-2500 \mathrm{~m}^{2}$ & 14,3 \\
\hline & $2501-5000 \mathrm{~m}^{2}$ & 37,5 \\
\hline
\end{tabular}

Fonte: Dados da Pesquisa.

O tamanho dos quintais variou, sendo a classe dominante entre $2501 \mathrm{~m}^{2}$ a $5000 \mathrm{~m}^{2}(37,5 \%)$ dos quintais, com idades entre 5 e 70 anos, todos delimitados por cercas de madeira e arame e circundados por vegetação nativa típica da fitofisionomia de Caatinga. Os cuidados que os mantenedores apresentam com seus quintais inclui a utilização de técnicas simples de manejo, como capinas periódicas, irrigação, limpeza e adubação. Estes cuidados, geralmente, envolvem toda a família, sobretudo as mulheres $(70 \%)$.

Dos 54 mantenedores/entrevistados, foi relacionada uma flora alimentícia de 70 espécies, distribuídas em 54 gêneros e 33 famílias (Tabela 2). A diversidade de Índice de Shannon-Wiener foi de 3,703, com média de 10,4 espécies por quintal. As famílias com maior número de espécies foram: Solanaceae (7 espécies; $10 \%$ das plantas encontradas), Anacardiaceae (6 espécies; 8,5\% das plantas encontradas), Annonaceae/Myrthaceae/ Rutaceae (4 espécies; 5,7\% das plantas encontradas) cada, as demais famílias $(64,4 \%)$ contribuíram com até três espécies cada. As espécies mais ocorrentes nos quintais foram: Anacardium occidentale L., Mangifera indica L. e Zea mays L.

Com relação ao hábito, foram observados quatro tipos: árvore, arbusto, herbáceo e subarbusto. Nos quintais estudados, houve a predominância do hábito arbóreo (32 espécies; 45,7\% dos hábitos encontrados) seguido por arbusto, erva (18 espécies cada; $25,7 \%$ dos hábitos encontrados) e pelo subarbustivo $(2 ; 2,9 \%)$.

Das espécies alimentícias cultivadas nos quintais, as frutíferas se sobressaíram em relação ao número de espécies (43 espécies; $61,5 \%$ das plantas encontradas), seguidas por hortaliças (12 espécies; 17,2\% das plantas encontradas) e espécies 
condimentares (15 espécies; $21,3 \%$ das plantas encontradas). As frutíferas mais encontradas nos quintais foram: $A$. occidentale; M. indica e Musa paradisiaca $\mathrm{L}$.

O cultivo de A. occidentale é bastante expressivo na comunidade, com 45 (71,4\%) dos quintais com cultivos dessa espécie, provavelmente devido à alta capacidade de comercialização local do seu fruto in natura, vendido para armazéns localizados no centro comercial da cidade, onde ocorre o beneficiamento da castanha. O pseudofruto é utilizado como ração para os animais. A maior parte da produção ocorre no período de setembro a novembro. Vale ressaltar que esta produção depende da quantidade de árvores presentes em cada quintal, podendo chegar até 400kg de castanha no período da safra.

Tabela 2. Plantas alimentícias cultivadas nos quintais da Comunidade Bom Lugar, Campo Maior/PI. Hábito: Arbusto (arb); Árvore (arv); Erva (erv); Subarbusto: Sub; Origem: Exótica (E); Nativa (N); PC: Parte comestível, Fo: Folha; Fr: Fruto; PF: Pseudofruto; Se: Semente; Ra: Raiz. Uso: C: Condimentar; F: Frutífera; H: Hortaliça;

\begin{tabular}{|c|c|c|c|c|c|}
\hline Família/Espécie & Nome Vulgar & Hábito & Origem & PC & Uso \\
\hline \multicolumn{6}{|l|}{ Aliaceae } \\
\hline Allium schoenoprasum $\mathrm{L}$. & Cebolinha & Erv & E & Fo & $\mathrm{H}$ \\
\hline \multicolumn{6}{|l|}{ Anacardiaceae } \\
\hline Anacardium occidentale $\mathrm{L}$. & Caju & Arv & $\mathrm{N}$ & $\mathrm{Fr}, \mathrm{PF}$ & $\mathrm{F}$ \\
\hline Mangifera indica $\mathrm{L}$. & Manga & Arv & E & Fr & $\mathrm{F}$ \\
\hline Spondias dulcis Parkinson & Cajarana & Arv & $\mathrm{E}$ & $\mathrm{Fr}$ & $\mathrm{F}$ \\
\hline Spondias mombin $\mathrm{L}$. & Cajá & Arv & $\mathrm{N}$ & $\mathrm{Fr}$ & $\mathrm{F}$ \\
\hline Spondias purpurea $\mathrm{L}$. & Siriguela & Arv & E & Fr & $\mathrm{F}$ \\
\hline Spondia tuberosa Arruda & Umbu & Arv & $\mathrm{N}$ & $\mathrm{Fr}$ & $\mathrm{F}$ \\
\hline \multicolumn{6}{|l|}{ Annonaceae } \\
\hline Anonna x atemoya Mabb. & Atemoia & Arv & E & $\mathrm{Fr}$ & $\mathrm{F}$ \\
\hline Annona coriacea Mart. & Araticum & Arv & $\mathrm{N}$ & $\mathrm{Fr}$ & $\mathrm{F}$ \\
\hline Annona muricata $\mathrm{L}$. & Graviola & Arv & $\mathrm{E}$ & $\mathrm{Fr}$ & $\mathrm{F}$ \\
\hline Annona squamosa $\mathrm{L}$. & Ata & Arb & E & $\mathrm{Fr}$ & $\mathrm{F}$ \\
\hline \multicolumn{6}{|l|}{ Apiaceae } \\
\hline Coriandrum sativum $\mathrm{L}$. & Coentro & Erv & E & Fo & $\mathrm{C}$ \\
\hline Petroselinum crispum (Mill.) Fuss. & Cheiro-verde & Erv & E & Fo & $\mathrm{C}$ \\
\hline \multicolumn{6}{|l|}{ Arecaceae } \\
\hline $\begin{array}{l}\text { Acrocomia aculeata (Jacq.) Lodd. ex } \\
\text { Mart. }\end{array}$ & Macaúba & Arv & $\mathrm{N}$ & $\mathrm{Fr}$ & $\mathrm{F}$ \\
\hline Cocos nucifera $\mathrm{L}$. & Coqueiro & Arv & E & $\mathrm{Fr}$ & $\mathrm{F}$ \\
\hline Euterpe precatoria Mart. & Açaí & Arv & $\mathrm{N}$ & Fr & $\mathrm{F}$ \\
\hline \multicolumn{6}{|l|}{ Asteraceae } \\
\hline Lactuca sativa $\mathrm{L}$. & Alface & Erv & $\mathrm{E}$ & Fo & $\mathrm{H}$ \\
\hline \multicolumn{6}{|l|}{ Bixaceae } \\
\hline Bixa orellana $\mathrm{L}$. & Urucum & Arb & $\mathrm{N}$ & $\mathrm{Se}$ & $\mathrm{C}$ \\
\hline \multicolumn{6}{|l|}{ Bromeliaceae } \\
\hline Ananas comosus (L.) Merril & Abacaxi & Erv & $\mathrm{N}$ & $\mathrm{Fr}$ & $\mathrm{F}$ \\
\hline \multicolumn{6}{|l|}{ Caricaceae } \\
\hline Carica papaya $\mathrm{L}$. & Mamão & Arb & E & $\mathrm{Fr}$ & $\mathrm{F}$ \\
\hline \multicolumn{6}{|l|}{ Cucurbitaceae } \\
\hline $\begin{array}{l}\text { Citrullus lanatus (Thunb.) Matsum. } \\
\& \text { Nakai }\end{array}$ & Melancia & Erv & $\mathrm{E}$ & Fr & $\mathrm{F}$ \\
\hline
\end{tabular}


Cucumis melo L.

Cucurbita pepo L.

Euphorbiaceae

Manihot esculenta Crantz

Ricinus communis $\mathrm{L}$.

\section{Fabaceae}

Arachis hypogaea $\mathrm{L}$.

Tamarindus indica $\mathrm{L}$.

Vigna unguiculata (L.) Walp.

\section{Lamiaceae}

Ocimum gratissimum $\mathrm{L}$.

\section{Lauraceae}

Persea americana Mill.

\section{Liliaceae}

Allium cepa L.

\section{Malpighiaceae}

Malpighia glabra L.

\section{Malvaceae}

Abelmoschus esculentus L. Moench

Hibiscus acetosella Welw. ex Hiern

Theobroma grandiflorum (Willd. ex Spreng.) K.Schum.

\section{Moraceae}

Artocarpus heterophyllus Lam.

Morus nigra L.

\section{Moringaceae}

Moringa oleifera Lam.

\section{Musaceae}

Musa paradisiaca $\mathrm{L}$.

\section{Myrthaceae}

Campomanesia velutina (Camb.) $\mathrm{O}$. Berg

Eugenia uniflora L.

Psidium guajava $\mathrm{L}$.

Syzygium cumini (L.) Skeels

\section{Oxalidaceae}

Averrhoa carambola L.

\section{Passifloraceae}

Passiflora sp.

Passiflora edulis Sims.

\section{Pedaliaceae}

Sesamum indicum $\mathrm{L}$.

\section{Poaceae}

Cymbopogon citratus (DC.) Stapf. Orysa sativa L.

Saccharum officinarum L.

\section{Melão}

Abóbora

Mandioca

Mamona

Amendoin

Tamarindo

Feijão

Alfavaca

Abacate

Cebola

Acerola

Quiabo

Vinagreira

Cupuaçu

Jaca

Amora

Moringa

Banana

Guabiraba

Pitanga

Goiaba

Azeitona-preta

Carambola

Maracujá-do-mato

Maracujá

Gergelim

Capim-de-cheiro

Arroz

Cana
Erv E

Erv E

Fr

F

Fr

$\mathrm{H}$

arb N

H

arb E

$\mathrm{H}$

Erv E

F

Arv E

Erv E

F

Arb E

Fo

C

Arv E

Fr

F

Erv

E

Fo

$\mathrm{H}$

Arv

E

Fr

F

Arb E

$\mathrm{Fr}$

$\mathrm{H}$

Arb

Arv

Fo

Fr

C

N

Arv E

$\mathrm{Fr}$

$\mathrm{Fr}$

Arv

E

Arv

E

Fr

F

Erv E

Fr

F

Arb N

Arb N

Arv N

Arv N

Fr

Fr

Fr

Fr

Arv

E

Fr

F

Arb $\quad$ - $\quad$ Fr

Arv N

$\mathrm{Fr}$

F

F

Arb N

Se

$\mathrm{H}$

Erv N

Fo

C

Erv

Fr

H

Erv

Fo

H 


\begin{tabular}{|c|c|c|c|c|c|}
\hline Zea mays $\mathrm{L}$. & Milho & Erv & $\mathrm{E}$ & Fr & $\mathrm{H}$ \\
\hline \multicolumn{6}{|l|}{ Rosaceae } \\
\hline Prunus avium (L.) L. & Cereja & Arv & $\mathrm{E}$ & $\mathrm{Fr}$ & $\mathrm{F}$ \\
\hline \multicolumn{6}{|l|}{ Rutaceae } \\
\hline Citrus $\times$ aurantium $\mathrm{L}$. & Laranja & Arv & $\mathrm{E}$ & $\mathrm{Fr}$ & $\mathrm{F}$ \\
\hline Citrus medica $\mathrm{L}$. & Limão-doce & Arv & $\mathrm{E}$ & Fr & $\mathrm{F}$ \\
\hline Citrus reticulata Blanco & Tangerina & Arv & $\mathrm{E}$ & Fr & $\mathrm{F}$ \\
\hline Citrus x limon (L.) Osbeck & Limão-azedo & Arv & $\mathrm{E}$ & Fr & $\mathrm{F}$ \\
\hline \multicolumn{6}{|l|}{ Sapindaceae } \\
\hline Talisia esculenta (Cambess.) Radlk & Pitomba & Arv & $\mathrm{N}$ & $\mathrm{Fr}$ & $\mathrm{F}$ \\
\hline \multicolumn{6}{|l|}{ Sapotaceae } \\
\hline Manilkara zapota (L.) P.Royen & Saputi & Arv & $\mathrm{E}$ & $\mathrm{Fr}$ & $\mathrm{F}$ \\
\hline Pouteria macrophylla (Lam.) Eyma & Tuturubá & Arv & $\mathrm{N}$ & $\mathrm{Fr}$ & $\mathrm{F}$ \\
\hline \multicolumn{6}{|l|}{ Solanaceae } \\
\hline Capsicum annuum $\mathrm{L}$. & Pimentão & Arb & $\mathrm{E}$ & $\mathrm{Fr}$ & $\mathrm{C}$ \\
\hline $\begin{array}{l}\text { Capsicum annuum var. glabrisculum } \\
\text { (Dunal) Heiser \& Pickersgiell }\end{array}$ & Pimenta-roxa & Arb & $\mathrm{N}$ & $\mathrm{Fr}$ & $\mathrm{C}$ \\
\hline Capsicum baccatum L. & Pimenta-calabresa & Arb & $\mathrm{N}$ & $\mathrm{Fr}$ & $\mathrm{C}$ \\
\hline $\begin{array}{l}\text { Capsicum baccatum var. pendulum } \\
\text { (Willd.) Eshbaugh }\end{array}$ & $\begin{array}{l}\text { Pimenta-dedo-de- } \\
\text { moça }\end{array}$ & Arb & $\mathrm{E}$ & $\mathrm{Fr}$ & $\mathrm{C}$ \\
\hline Capsicum chinense Jacq. & Pimenta-de-cheiro & Arb & $\mathrm{N}$ & Fr & $\mathrm{C}$ \\
\hline Capsicum frutescens $\mathrm{L}$. & Pimenta-malagueta & Arb & $\mathrm{N}$ & $\mathrm{Fr}$ & $\mathrm{C}$ \\
\hline Solanum lycopersicum L. & Tomate & Erv & $\mathrm{E}$ & $\mathrm{Fr}$ & $\mathrm{C}$ \\
\hline \multicolumn{6}{|l|}{ Verbenaceae } \\
\hline $\begin{array}{l}\text { Lippia alba (Mill.) N.E. Br. ex } \\
\text { Britton \& P. Wilson }\end{array}$ & Erva-cidreira & Sub & $\mathrm{N}$ & Fo & $\mathrm{C}$ \\
\hline Vitex flavens Humb., Bonpl. \& Kunth & Mama-cachorra & Arv & $\mathrm{N}$ & Fr & $\mathrm{F}$ \\
\hline \multicolumn{6}{|l|}{ Vittaceae } \\
\hline Vittis vinifera $\mathrm{L}$. & Uva & Arb & $\mathrm{E}$ & Fr & $\mathrm{F}$ \\
\hline \multicolumn{6}{|l|}{ Zingiberaceae } \\
\hline Curcuma longa $\mathrm{L}$. & Açafrão & Erv & $\mathrm{E}$ & $\mathrm{Ra}$ & $\mathrm{C}$ \\
\hline
\end{tabular}

Fonte: Dados da Pesquisa.

Com relação a ocorrência nos quintais, as frutíferas também se sobressaíram, pois ocorreram em todos os quintais (54 dos quintais; $100 \%$ dos quintais amostrados), seguidas pelas hortaliças $(45 ; 71,4 \%)$ e condimentares $(18 ; 33,3 \%)$. O cultivo de hortaliças e condimentares é realizado em canteiros suspensos (Figura 2), o que demanda pouco espaço no quintal e a maior parte destas espécies são produzidas $(92,8 \%)$ é mantida para o autoconsumo. Em 8,2\% dos quintais, foi observada a comercialização das hortaliças e condimentares, sendo estas vendidas na própria comunidade e/ou no mercado público da cidade.

Além dessas, outra espécie que possui status comercial é a Manihot esculenta Crantz (mandioca), cultivada em 16 quintais (29,6\%). Em épocas de safra, os moradores se organizam e produzem farinha, bem como outros produtos derivados de mandioca nas casas de farinhas, presentes nos quintais da comunidade. 
Figura 2. Canteiro suspenso encontrado nos quintais de Bom Lugar, Campo Maior-PI.

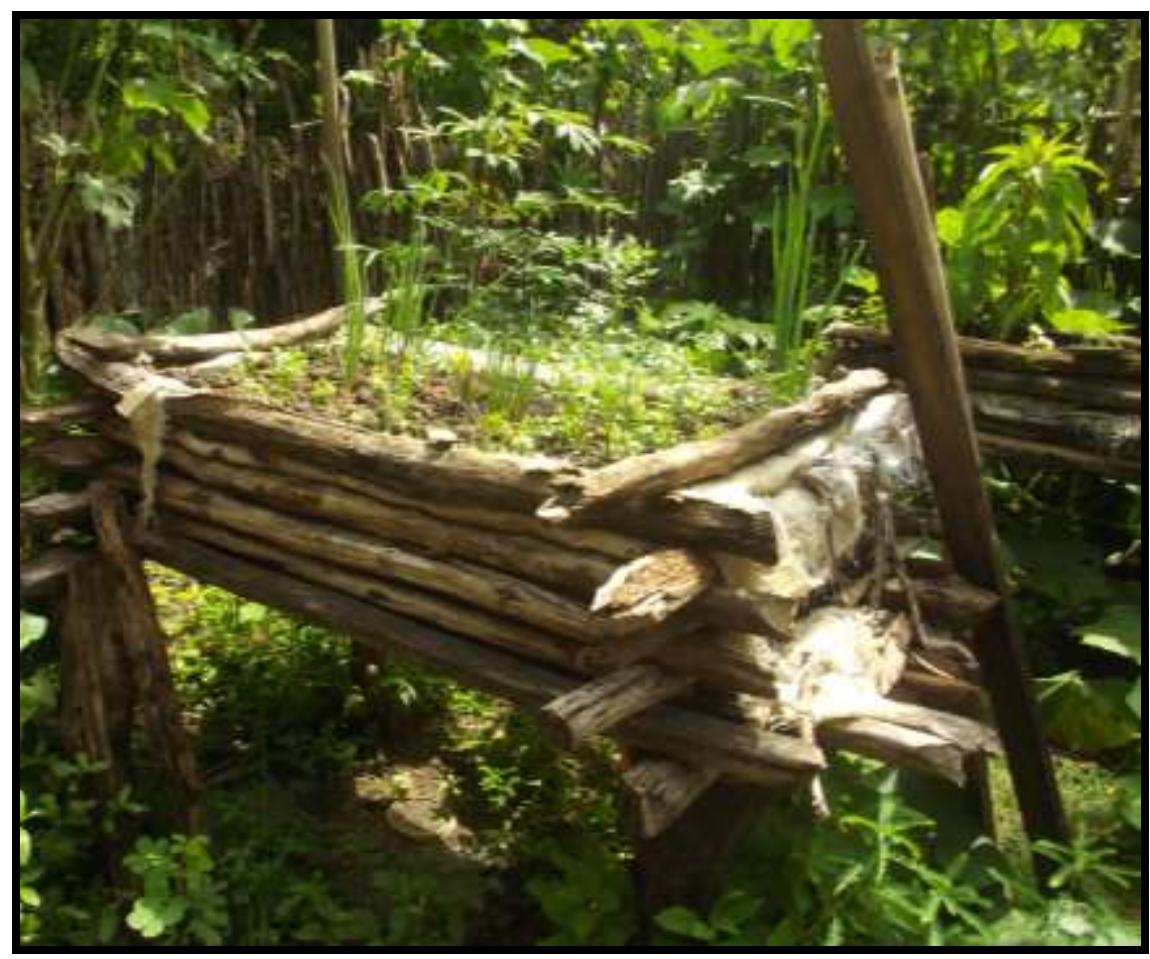

Fonte: Autores.

Sobre a origem das plantas encontradas, o número de exóticas $(44 ; 62,8 \%)$ foi superior ao número de nativas (26; 37,2\%). Das plantas exóticas, as de maior ocorrência nos quintais foram $M$. indica (39 quintais; 69,6\% dos quintais amostrados) e $M$. glabra (acerola) (29 quintais; 53,7\% dos quintais amostrados). Destaca-se que $M$. indica, além do uso alimentício, a maior presença desta espécie foi relatada por conta do seu uso como ornamental e por proporcionar sombra aos quintais. Dentre as espécies nativas, A. occidentale (caju) (45 quintais, 71,4\%) e P. guajava (goiaba) (24 quintais, 44,4\%) aparecem como as mais encontradas.

Cerca de 39\% (21) das plantas alimentícias encontradas nos quintais são consumidas na forma de chás e foram relatadas como medicinais, com destaque para as espécies condimentares, erva-cidreira ( $L$. alba) e capim-de-cheiro $(C$. citratus) e frutíferas como o limão (Citrus x limon), sendo estas, espécies mais encontradas nos quintais.

\section{Discussão}

A maior parte dos quintais estudados é gerida por mulheres, este fato reforça o que Pimbert (2009) afirma: as mulheres rurais são a maior força de trabalho dos sistemas alimentares e contribuem de maneira significativa para a segurança alimentar e para a economia local.

Destaca-se também que as mulheres têm papel fundamental na manutenção dos quintais, pois, ao realizarem o processo de experimentação e seleção das espécies, estas contribuem para o incremento da diversidade nestes ambientes. Oakley (2004) afirma que as mulheres, ao utilizarem espécies pouco conhecidas ou subutilizadas, preservam a biodiversidade, transformando seus quintais em ambientes de manipulação e de conhecimento para a adaptação de variedades locais e espécies não domesticadas.

A expressiva quantidade de plantas alimentícias encontradas nos quintais em nosso estudo era esperada, uma vez que estudos realizados em quintais têm revelado a predominância desse grupo (Florentino, Araújo \& Albuquerque, 2007; Botelho, Lamano-Ferreira \& Ferreira, 2014; Ramli, Milow \& Malek, 2021). Destaca-se também que a composição de espécies 
encontradas nos quintais de Bom lugar demonstra que estes sistemas produtivos são bastante diversificados. Para Oliveira (2006), essa diversificação tem grande importância para os mantenedores dos quintais, pois permitem a produção ao longo de todo ano.

A diversidade de Shannon encontrada nos quintais de Bom Lugar foi semelhante aos achados de Pereira (2016), este autor encontrou a diversidade de $\left(H^{\prime}=3,45\right)$ em quintais de comunidades rurais no município de Monsenhor Gil/PI. Entretanto, estudos realizados em outras localidades obtiveram uma diversidade menor, a exemplo de Pilla e Amoroso (2008) estudando quintais em bairros rurais no estado de São Paulo $\left(H^{\prime}=1,98\right)$ e Barreira et al. (2015) na zona rural de Minas Gerais $\left(H^{\prime}=1,65\right)$, este fato sugere que os quintais presentes nas áreas rurais piauienses apresentam elevada diversidade vegetal.

A família Solanaceae, mais representativa em número de espécies neste estudo, é uma das famílias de angiospermas de alto valor econômico em todo o mundo, pois possuem tanto espécies alimentícias de alto rendimento quanto plantas medicinais, apresentando papel central na nutrição humana desde o início da civilização humana (Ghatack et al., 2017; Alfroz et al., 2020).

Sobre o hábito, as árvores foram predominantes, resultados semelhantes foram encontrados por Nascimento et al. (2015) ao estudarem as plantas alimentícias espontâneas no município de Barreira, Bahia. A maior ocorrência do hábito arbóreo tem sido relatada em estudos realizados em quintais (Kumar, 2006) por apresentarem várias finalidades, como: alimento, sombra, madeira e outros, fornecendo vários serviços para os mantenedores dos quintais (Duque-Brasil et al., 2011).

As frutíferas foram o grupo mais encontrados nos quintais estudados, destaca-se que o cultivo dessas plantas é bastante comum nos quintais (Semedo \& Barbosa, 2007; Florentino, Araújo \& Albuquerque, 2017; Whitney et al. 2018; Mathewos, Hundera \& Biber-Freundenberger, 2018), pois o cultivo desse grupo promove a subsistência das famílias e contribui para a conservação da biodiversidade, fornecendo suplementação alimentar às famílias e permitindo às comunidades reduzirem a dependência de produtos advindos de locais externos (Pasa, 2004; Nascimento et al., 2015; Mathewos, Hundera \& Biber-Freundenberger, 2018).

Dentre as plantas de maior importância nos quintais, a presença expressiva de A. occidentale (caju) nos quintais estudados pode estar relacionada ao seu alto valor econômico. Esta é uma espécie bastante comum no Brasil e a região Nordeste tem se destacado no cenário nacional, sobretudo, na região do semiárido, por gerar postos de trabalho e renda na época mais seca do ano (Brainer \& Vidal, 2018; Novaes \& Novaes, 2021). E em quintais, em virtude das variadas possibilidades de consumo desta espécie tem sido relatada como de maior ocorrência (Florentino, Araújo \& Albuquerque, 2007).

O beneficiamento da mandioca (M. esculenta), por meio das casas de farinhas, consiste em uma prática comum no Nordeste brasileiro, demonstrando o envolvimento de toda a comunidade, realizado comumente nos quintais, revelando assim a importância destes, enquanto espaço de tradição e socialização. Estudos realizados em quintais têm demonstrado que estes são espaços de múltiplos significados, tanto para vida individual quanto coletiva, configurando locais de lazer, socialização, lugar de tradição, cultura e interação familiares (Tourinho \& Silva, 2016; Lobato et al., 2017).

Evidencia-se que o cultivo de hortaliças e condimentares nos quintais amostrados se apresenta como uma forma de estratégia para manter a autonomia no fornecimento dessas espécies frescas. A produção agrícola para autoconsumo pode ser considerada uma importante fonte de segurança alimentar na comunidade estudada, isto porque mais de $90 \%$ do que é produzido nestes quintais são destinados para os mantenedores dos quintais. Fato semelhante foi encontrado por Garcia, Vieira \& Oliveira (2015), ao avaliarem a contribuição dos quintais agroflorestais na Amazonia oriental, onde verificaram que a maior parte do que é produzido era utilizado para o autoconsumo, demonstrando que estes sistemas possibilitam acesso fácil, rápido e direto a uma variedade de alimentos, fontes de nutrientes essenciais a dieta humana. 
Ao analisar a origem das plantas alimentícias encontradas nos quintais, as exóticas apresentaram maior número do que as nativas. Resultados semelhantes foram encontrados por Silva, Oliveira \& Abreu (2017) estudando quintais de uma comunidade rural no Piaú e por Duque-Brasil (2011) analisando a diversidade de espécies arbóreas em quintais rurais de Minas Gerais. Albuquerque, Cavalcanti \& Cabarello (2005) afirmam que é comum a predominância de espécies exóticas em quintais.

Destaca-se que o impacto das plantas exóticas no ambiente natural tem sido bastante discutido (Marco et al., 2008). Em quintais, estas espécies apresentam um importante papel, pois contribuem para o incremento da diversidade vegetal ao atenderem as demandas de uso dos mantenedores (Duque-Brasil et al., 2011). Embora a presença de nativas seja menor do que a de exóticas nos quintais de Bom Lugar, a presença de nativas pode ser um indicativo que os quintais estudados contribuem para a conservação da biodiversidade local. O cultivo de espécies nativas em quintais garante a variabilidade genética, constituindo um importante banco de germoplasma (Brito \& Coelho, 2000).

Um número considerável das plantas encontradas neste estudo foi utilizado como medicamentos, isto era esperado, pois é comum encontrar plantas alimentícias utilizadas como medicinais em quintais (Freitas et al., 2015). Por exemplo, na Tailândia, Panyadee et al (2019), estudando as plantas medicinais em quintais de quatro grupos étnicos, encontraram que a maior parte das plantas medicinais presentes nos quintais tinham como uso primário a alimentação, revelando assim a estreita relação entre a alimentação local e a medicina na vida dessas etnias. Mendes et al. (2019) afirmam que as propriedades medicinais das plantas utilizadas como alimento são comumente subentendidas no seu contexto alimentar, sendo uma oportunidade para prevenir doenças. Destaca-se também que, por serem os usos culturais mais expressivos, as plantas de uso alimentício e medicinal são mantidas em taxas altas, mesmo em sociedades industrializadas (Gras et al., 2016).

\section{Considerações Finais}

A diversidade de plantas alimentícias encontradas nos quintais pesquisados revela como esses sistemas produtivos são diversificados e contribuem para a nutrição e subsistência dessas famílias, sobretudo nas áreas rurais, que são as mais afetadas com a insegurança alimentar.

Embora a maior parte das espécies alimentícias encontradas nos quintais tenha como intuito a utilização para o autoconsumo, as espécies utilizadas como fonte de renda, contribuíram de maneira significativa para os mantenedores, considerando o baixo poder aquisitivo dessas populações.

A presença de plantas nativas encontradas reforça o papel dos quintais como locus da biodiversidade, diminuindo a pressão sobre as florestas naturais. Ressalta-se, também, que as atividades realizadas nos quintais refletem os múltiplos significados dos quintais, enquanto espaços de lazer, socialização de conhecimentos e práticas culturais.

\section{Referências}

Afroz, M., Akter, S., Ahmed, A. Rouf, R., Shilpi, J. A., Tiralongo, E., Sarker, S. D., Gorasson, U., \& Uddin, S. J. (2020). Ethnobotany and Antimicrobial Peptides From Plants of the Solanaceae Family: An Update and Future Prospects. Front Pharmacology, 11 (565), 1-15.

Aguiar, L. C. G. G., \& Barros, R. F. M. (2012). Plantas Medicinais cultivadas em quintais de comunidades rurais no domínio do Cerrado piauiense (Município de Demerval Lobão, Piauí, Brasil). Revista Brasileira de Plantas Medicinais, 14 (3), 419-434.

Albuquerque, U. P., Ramos, M. A., Lucena, R. F. P, \& Alencar, N. L. (2014). Methods and techniques used to collect ethnobiological data. (2, 15- 37), Springer.

Albuquerque, U. P., Cavalcanti, L. H., \& Caballero, J. (2005). Structure and Floristics of Homegardens in Northeastern Brazil. Journal of Arid Environments, 62(3), 491-506.

APG IV. (2016) An update of the Angiosperm Phylogeny Group classification for the orders and families of flowering plants: APG IV. Botanical Journal of the Linnean Society, 181, 1-20. 
Amorim, N. A. (2015). Quintais urbanos em Teresina (PI): cultivo de espécies úteis e apego ao lugar. 2015. 99f. Tese (Doutorado em Desenvolvimento e Meio Ambiente) - Universidade Federal do Piauí, Teresina.

Barbosa, A. R. (2007). Os humanos e os répteis da mata: uma abordagem etnoecológica de São José da Mata - PARAíBA. 2007.123 f. Dissertação (Desenvolvimento e Meio Ambiente) - Universidade Federal da Paraíba, João Pessoa.

Barreira, T. F., Paula Filho, G. X., Rodrigues, V.C.C., Andrade, F. M. C., Santos, R. H. S., Priore, S. E., \& Pinheiro-Sant'ana, H. M. (2015) Diversidade e equitabilidade de plantas alimentícias não-convencionais na xona rural de Viçosa, Minas Gerais, Brasil. Revista Brasileira de Plantas Medicinais, 17(4), 964974.

Batista Filho, M. (2010). Direito à alimentação. Revista Brasileira de Saúde Materno Infantil. 10 (2), 1-2.

Bernard, H. R. (1998). Research Methods in Cultural Antrhropology, Sage, Newbury Park, CA, EUU.

Brainer, M. S. C. P., \& Vidal, M. F. (2018). Cajucultura Nordestina em recuperação. Caderno Setorial Etene, 3(54), 1-13.

Brasil. Constituição (1988). Constituição da República Federativa do Brasil. Senado Federal: Centro Gráfico.

Brito, M. M.; Coelho, M. F. (2000). Os Quintais Agroflorestais em Regiões Tropicais - Unidades Auto Sustentáveis. Agricultura Tropical, 4 (1), $7-38$.

Botelho, J. M., Lamano-Ferreira, A. P. N., \& Ferreira, M. L. (2014). Cultivation and use of domestic plants in different Brazilian cities. Ciência Rural, 44 (10), $1810-1815$.

Calvet-Mir, L., March, H., Corbacho-Monne, D., Gomez-Baggethun, E., \& Reyes-Garcia, V. (2016). Home Garden Ecosystem Services Valuation through a Gender Lens: A Case Study in the Catalan Pyrenees. Sustainability, 8 (718), https://doi.org/10.3390/su8080718.

Carneiro, M. G. R., Machado, A. C., Esmeraldo, G. G. S. L, \& Sousa, N. R. (2013). Quintais Produtivos: contribuição à segurança alimentar e ao desenvolvimento sustentável local na perspectiva da agricultura familiar (O caso do Assentamento Alegre, município de Quixeramobim/CE). Revista Brasileira de Agroecologia, 8, (2), 135-147.

Castañeda-Navarrete, J. (2021). Homegarden diversity and food security in southern Mexico. Food Security. https://doiorg.ez17.periodicos.capes.gov.br/10.1007/s12571-021-01148-w.

Duque-Brasil, R.., Soldati, G. T., Espirito-Santo, M. M., Rezende, M. Q., D’Ângelo-Neto, S., \& Coelho, F. M. G. (2012). Composição, uso e conservação de espécies arbóreas em quintais de agricultores familiares na região da mata seca norte-mineira, Brasil. SITIENTIBUS série Ciências Biológicas, 11 (2), 287 , 2012.

Farias, R. R. S., \& Castro, A. A. J. F. (2004) Fitossociologia de trechos da vegetação do complexo de campo maior, Campo Maior, PI, Brasil. Acta Botanica Brasilica, 18 (4), 949-963.

Flora do Brasil 2020 em construção. (2020). Jardim Botânico do Rio de Janeiro. http://floradobrasil.jbrj.gov.br/.

Florentino, A. T. N., Araújo, E. L., Albuquerque, U.P. (2007). Contribuição de quintais agroflorestais na conservação de plantas da Caatinga, Município de Caruaru, PE, Brasil. Acta Botânica Brasilica. 21 (1), 37-47.

Food and Agriculture Organization of The United Nations - FAO. (2019). The State of Food and Agriculture 2019. Moving forward on food loss and waste reduction. Rome.

FAO. (2015). Declaração de Roma sobre a segurança alimentar mundial e Plano de ação da cúpula mundial da alimentação. http://www.fao.org/ docrep/003/w3613p/w3613p00.HTM\#Note1.

FAO-BRASIL (2015). O Estado da Segurança Alimentar e nutricional no Brasil 2015. http://www.fao.org/fileadmin/user_upload/FAOcountries/Brasil/docs/SOFI_Brasil_2015_final.pdf.

Freitas, A.V., Coelho, M. F. B., Pereira, Y. B., Freitas Neto, E. C. A., \& Azevedo, R. A. B. Diversidade e usos de plantas medicinais nos quintais da comunidade de São João da Várzea em Mossoró, RN. Revista Brasileira de Plantas Medicinais, 17 (4), 845-856.

Garcia, B. N. R., Vieira, T. A., \& Oliveira, F. A. Quintais agroflorestais e segurança alimentar em uma comunidade rural na Amazônia Oriental. Revista de la Facultad de Agronomía, 114(1), 67-73.

Galluzzi, G., Eyzaguirre, P., \& Negri, V. (2010). Home gardens: Neglected hotspots of agro-biodiversity and cultural diversity. Biodiversity and Conservation 19: 3635-3654.

Gazel Filho, A. B., Yared, J. A. G., Mourão Júnior, M., Cordeiro, I. M. C. C., \& Brienza Júnior, S. (2009). Contribuição de quintais agroflorestais para a segurança Alimentar em Mazagão, AP. In: Congresso Brasileiro de Sistemas Agroflorestais, 7, 2009. Luziânia, GO. Anais. EMATER, EMBRAPA.

Ghatak, A., Chaturvedi, P., Paul, P., Agrawal, G. K., Rakwla, R., Kim, S. T., Weckwerth, W., \& Gupta, R. (2017). Proteomics survey of Solanaceae family: Current status and challenges ahead. Journal of Proteomics. 3(169), 41-57.

Gras, A., Garnatje, T., Bonet, M. A., Carrió, E., Mayans, M. Parada, M., Rigat, M., \& Vallés, J. (2016). Beyond food and medicine, but necessary for life, too: other folk plant uses in several territories of Catalonia and the Balearic Islands. Journal of Ethnobiology and Ethnomedicine. 12 (23), 1-53.

Instituto Brasileiro de Geografia e Estatística (IBGE) (2010). Censos demográficos. http://www.ibge.gov.br/home/estatistica/populacao/censo2010/.

IBGE. (2014). Pesquisa Nacional por Domicílios: Segurança Alimentar 2013. Coordenação de Trabalho e Rendimento.

IBGE. (2020). Pesquisa de Orçamentos Familiares 2017-2018: análise da segurança alimentar no Brasil. Coordenação de Trabalho e Rendimento. 
Kamiyama, C., Hashimoto, S., Kohsaka, R., \& Saito, O. (2016). Non-market food provisioning services via homegardens and communal sharing in satoyama socio-ecological production landscapes on Japan's Noto península. Ecosystem Services, 17, 185-196.

Kumar, B. M. (2006). Carbon sequestration potential of tropical homegardens. (185-204), Springer.

Kumar, B. M., \& Nair, P. (2004). Ramachandran. The enigma of tropical homegardens. Agroforestry Systems, 61 (1), $135-152$.

Lobato, G.J.M., Lucas, F. C. A., Tavares-Martins, A.C. C., Jardim, M. A. G., \& Martorano, L.G. (2017). Diversidade de uso e aspectos socioambientais de quintais urbanos em Abaetuba, Pará, Brasil. Revista Brasileira de Agroecologia. 12, (3), 95-105.

Maas, N. M., Mendoza-Sassi, R. A., Meucci, R. D., \& Cesar, J. A. (2020). Food insecurity in rural families in the extreme south of Brazil. Ciência e Saúde Coletiva. 25 (7), 2605-2614.

Marco, A., Dutoit, T., Deschamps-Cottin, M., Mauffrey, J., Vennetier, M.; \& Bertaudiére-Montes, V. (2008). Gardens in urbanizing rural areas reveal an unexpected floraldiversity related to housing density. Ecology, 331, 452-465.

Magurran, A. E. (2013) Measuring Biological Diversity. Tradução: Vianna, DM. Medindo a Diversidade Biológica. UFPR.

Mathewos, W., Hundera, K., \& Biber-Freudenberger, L. (2018). Planting Fruits and Vegetables in Homegarden as a Way to Improve Livelihoods and Conserve Plant Biodiversity. Agriculture,. 8 (12), 1-17. https://doi.org/10.3390/agriculture8120190

Mendes, R. B. E., Carvalho, M. A. N., Santos, P. S., Pereira, M. F., Anjos, A. V. M., \& Malta Junior, A. (2019). Investigação do conhecimento tradicional e científico de plantas alimentícias em Juazeiro do Norte-CE-BRA. Revista E-Ciência, 7(2), 12-16.

Mohri, H., Lahoti, S., Saito, O., \& Herath, S. (2013) Assessment of ecosystem services in homegarden systems in Indonesia, Sri Lanka, and Vietnam. Ecosystem Services. 5, 124-136.

Nascimento, V. T., Pereira, H. C., Silva, A. S., Nunes, A. T., \& Medeiros, P. M. (2015). Plantas alimentícias espontâneas conhecidas pelos moradores do Vau da Boa Esperança, Município de Barreiras, Oeste da Bahia, Nordeste do Brasil. Revista Ouricuri, 5(1), 86-109.

Nascimento, A. P. B. do, Alves, M. C., \& Molina, S. M. G. (2005). Quintais domésticos e sua relação com estado nutricional de crianças rurais, migrantes e urbanas. Multiciência, http://www.multiciencia.unicamp.br/rede_3_5.htm.

Nações Unidas (2014) Documento Final - Grupo de Trabalho Aberto sobre Objetivos de Desenvolvimento Sustentável.

Novaes, T. E. R., Novaes, A. S. R. (2021). Análise dos potenciais medicinais do cajueiro (Anacardium occidentale Linn): uma breve revisão. Research, Society and Development, $10(1), 1-7$.

Oakley, E. (2004). Quintais Domésticos: uma responsabilidade cultural. Agriculturas, 1, (1), 37-39.

Oliveira J. S. R. (2006). Uso do Território, Experiências Inovadoras e Sustentabilidade: um estudo em Unidades de Produção Familiares de agricultores na área de abrangência do Programa PROAMBIENTE, Nordeste Paraense. 2006. 110f. Dissertação (Mestrado em Agriculturas Amazônicas) - Universidade Federal do Pará, Pará

Panydaee, P.. Balslev, H., Wangpakattanawong, P., \& Inta, A. (2019). Medicinal plants in homegardens of four ethnic groups in Thailand. Journal of Ethnopharmacology. 239(15), 1-14. https://doi.org/10.1016/j.jep.2019.111927

Pasa, M. C. (2004). Etnobiologia de uma comunidade ribeirinha no alto da bacia do rio Aricá-Açú, Cuiabá, Mato Grosso, Brasil. 2004. 174 f. Tese (Doutorado em Ciências Biológicas) - Universidade Federal de São Carlos, São Carlos.

Pereira, L. G. (2016). Quintais rurais: etnobotânica com enfoque na diversidade e uso de plantas no município de Monsenhor Gil, Piauí, Nordeste do Brasil. 2016. 136f. Tese (Doutorado em Desenvolvimento e Meio Ambiente) - Universidade Federal do Piauí, Teresina.

Pereira, L. G., Vieira, F. J., Alencar, N. L., Carvalho, F. P. A., \& Barros, R. F. M. (2016). Diversidade florística em quintais do Nordeste brasileiro: Um estudo etnobotânico em comunidades rurais em Monsenhor Gil/PI. Espacios, 37 (11), 1-11.

Pilla, M. A. C., \& Amorozo, M. C. M. (2009). O conhecimento sobre os recursos vegetais alimentares em bairros rurais no Vale do Paraíba, SP, Brasil. Acta Botânica Brasílica. 23(4), 1190-1201.

Pimbert, M. P. (2009). Mulheres e soberania alimentar. In: Mulheres construindo a Agroecologia. Revista Agriculturas, 6 (4), $41-45$.

Ramli, M. R., Milow, P., \& Malek, S. (2021). Species composition, diversity and traditional uses of plants in homegardens in Kampung Masjid Ijok, Perak, Malaysia. Biodiversitas, 22 (4), 1902-1911. 10.13057/biodiv/d220438

Ribeiro, K. V., Amorim, N. A., \& Barros; R. F.M. (2020). Composição, Uso Potencial e Gerenciamento de Recursos Vegetais em um Tipo de Sistema Agroflorestal como Estratégia de Manutenção e Conservação. Fronteiras, 9(2), 348-370.

Semedo, R. J. C. G., \& Barbosa, R. I. (2007). Árvores frutíferas nos quintais urbanos de Boa Vista, Roraima, Amazônia brasileira. Acta Amazonica, 37(4), 497-504.

Silva, P. H., Oliveira, Y. R., \& Abreu, M. C. (2017). Uma abordagem etnobotânica acerca das plantas úteis cultivadas em quintais em uma comunidade rural do semiárido piauiense, Nordeste do Brasil. Journal of Environmental Analysis and Progress, 6(2), 144-159.

Team, R. 2018 Core. R: A Language and Environment for Statistical Computing. 
Research, Society and Development, v. 10, n. 6, e24510615628, 2021

(CC BY 4.0) | ISSN 2525-3409 | DOI: http://dx.doi.org/10.33448/rsd-v10i6.15628

Tourinho, H. L. Z., \& Silva, M. G. A. (2016). Quintais urbanos: funções e papéis na casa brasileira e amazônica. Boletim do Museu Paraense Emílio Goeldi. Ciências Humanas, 1.11(3), 633-65.

Velloso, A. L., Sampaio, E. V.S. B., \& Pareyn, F. G. C. (2002). Ecorregiões propostas para o Bioma Caatinga. PNE.

Whitney, C. W., Luedeling, E., Hensel, O., Tabuti, J.R.S., Krawinkel, M., Gebauer, J., \& Kehlenbeck, K. (2018). The Role of Homegardens for Food and Nutrition Security in Uganda. Human Ecology, 46, 497-514.

Zhang, Y., Yang, L., \& Wang, Y. (2020). The best choices: the diversity and functions of the plants in the home gardens of the Tsang-la (Motuo Menba) communities in Yarlung Tsangpo Grand Canyon, Southwest China. Journal of Ethnobiology and Ethnomedicine. 16, 16-50. 